\title{
Quelques facteurs déterminant le comportement de butinage d'Hypotrigona pothieri (Trigonini) dans la végétation de Côte-d'Ivoire
}

\author{
D Lobreau-Callen 1*, A Le Thomas 2, B Darchen 3, R Darchen 3 \\ avec la collaboration technique de N D'Amico 2
}

' CNRS et EPHE, Laboratoire de phanérogamie du Muséum, 16, rue Buffon, 75005 Paris;

2 Laboratoire de phytomorphologie de l'EPHE, 16, rue Buffon 75005 Paris;

${ }^{3}$ Station biologique de l'université de Paris-VI, 24620 Les Eyzies, France

(Reçu le 3 mai 1989; accepté le 20 décembre 1989)

\begin{abstract}
Résumé - L'analyse pollinique des récoltes d'Hypotrigona pothieri Darchen (Hym., Apidae) est réalisée sur 7 ruches transplantées dans des biotopes différents et comparée à celle d'une ruche témoin de la savane de Lamto, après 5 mois d'installation. Les résultats montrent que, malgré un spectre pollinique global assez étendu, ces abeilles sociales, de caractère généraliste et opportuniste, sont relativement sélectives. Elles privilégient un petit nombre d'espèces végétales toujours proches de leurs nids, indépendamment du type de structure florale mais, semble-t-il, en fonction de la présence de certains alcaloïdes dans les nectars. Leur choix est directement influencé par l'orientation de l'ouverture des nids, et la lumière est indispensable à leur survie.
\end{abstract}

\section{Hypotrigona pothieri / comportement de butinage / analyse pollinique / écologie / Apidae}

\section{INTRODUCTION}

Dans les régions tropicales, les abeilles sociales sans dard (Apidae, Meliponinae) représentent un groupe très important de pollinisateurs et de récolteurs de nectar. Depuis une dizaine d'années, leur biologie et leur écologie ont été assez bien étudiées dans les néotropiques, en particulier dans les forêts du Costa Rica et de Panama où elles sont très abondantes (Johnson et Hubbell, 1974, 1975; Hubbell et Johnson, 1977, 1978; Roubik, 1978, 1979, 1980, 1981, 1982; Roubik et al, 1986) ou encore dans des zones fortement anthropisées du Brésil (Kleinert-Giovannini et Imperatriz-Fonseca, 1987; Cortopassi-Laurino et Ramalho, 1988). Les inventaires sur les ressources polliniques de quelques espèces (Absy et Kerr, 1977; Wille et Orozco, 1975; Absy et al, 1980; Iwama et Melhem, 1979; Engel et DingemansBakels, 1980) font apparaître qu'elles sont très généralistes, la diversité des fleurs visitées étant d'autant plus grande que le nombre d'insectes constituant le nid est important (Sommeijer et al, 1983). Ceci rejoint les conclusions de Roubik (1979) sur

\footnotetext{
* Correspondance et tirés à part.
} 
la compétitivité des abeilles sociales sans dard chez lesquelles il constate que les Trigona sl sont les plus généralistes et plus spécialement Melipona fulva, comparable de ce point de vue à Apis mellifera var adansonii, abeille africaine introduite. Cependant, à la Trinité, Sommeijer et al (1983) ont montré que, malgré un recouvrement considérable des pollens récoltés par plusieurs espèces de Mélipones, et un butinage continu de certains types de pollens, chacune des espèces visite les fleurs selon un schéma sélectif et uniforme, corrélé avec la taille de la population de la coIonie.

Sur le continent africain, le comportement des abeilles sociales sans dard a été beaucoup moins étudié. L'éthologie de la tribu des Trigonini (Darchen, 1985) et certains aspects de leur action sur la végétation ont été abordés par l'analyse pollinique de leurs récoltes (Lobreau-Callen et Callen, 1986; Le Thomas et al, 1988). On a pu montrer, en particulier, que dans une même savane, le recouvrement des ressources polliniques de trois espèces de Trigona sl est beaucoup plus restreint que celui qui a été observé à la Trinité par Sommeijer et al (1983) et que les différences dans la composition des récoltes polliniques de chacune d'elles indiquent des stratégies de butinage spécifiques, dans le choix des espèces privilégiées, cómme dans les strates visitées. Par ailleurs, comme le soulignent Frankie et al (1983), le butinage intense de certaines espèces n'indiquerait pas nécessairement une action pollinisatrice.

Dans ce travail, nous examinerons plus particulièrement le comportement d'une même espèce d'Hypotrigona, genre endémique d'Afrique : $H$ pothieri, originaire de la savane et transplantée dans différents biotopes de la même région. Nous dégagerons, à travers ses récoltes polliniques, certaines de ses exigences et son action sur la végétation environnante.

\section{LE MILIEU}

Hypotrigona pothieri affine de $H$ gribodoi Mayr (Darchen, 1985) est une espèce très commune dans la savane de Lamto, située à $200 \mathrm{~km}$ au nord d'Abidjan en Côted'Ivoire. Cette savane, incluse dans les galeries forestières longeant le fleuve Bandama, est caractérisée par une strate graminéenne à Imperata cylindrica. Loudetia simplex, Andropogon spp, Hyparrhenia spp et un peuplement de Borassus aethiopicum qui domine la strate arbustive constituée essentiellement de Crossopteryx febrifuga, Piliostigma thonningii, Cussonia barteri, Bridelia ferruginea et Annona senegalensis (Menaut, 1973; César et Menaut, 1974; Schmidt, 1973; Lamotte, 1978; Vuattoux, 1976; Jaffre et Namur, 1983; Devineau et al, 1984).

$H$ pothieri est une espèce de petite taille, environ celle d'un moucheron, qui établit ses nids dans le tronc ou les grosses branches de Bridelia ferruginea. Crossopteryx febrifuga, Piliosptigma thonningii, Terminalia glaucescens ou Vitex doniana. Curieusement, on remarque qu'aucun nid n'est édifié dans Cussonia barteri pourtant très abondant dans cette savane, peut-être en raison de la structure spongieuse du bois.

\section{MÉTHODES ET SITES D'ÉTUDE}

Au cours du mois de mars 1985, correspondant à la saison des pluies pendant laquelle la diversité florale est importante, 7 nids d'Hypotrigones ont été prélevés dans la savane, dans la région de Zougoussi, village situé à environ $6 \mathrm{~km}$ au nord de la station biologique de Lamto. Ils ont alors été transplantés dans 5 sites différents (R1 à R5) correspondant respectivement à un biotope précis dont les espèces végétales 
les plus proches sont recensées dans le tableau I. Un rucher témoin RT est resté dans son lieu d'origine pour établir les comparaisons avec les récoltes effectuées dans les différents nids ouverts en août 1985, c'est-à-dire à la fin de la petite saison sèche, soit environ 5 mois après le début de l'expérience:
- R1: un nid placé entre 2 branches de Lasiodiscus mildbraedii, au bord d'un chemin conduisant au fleuve Bandama dans la galerie forestière;

- R2: un nid situé en milieu ouvert, à la lisière de la savane et de la galerie forestière, dans un fourré de Cussonia barteri et Piliostigma thonningii;

Tableau I. Composition floristique des différents sites d'implantation des ruchers d'Hypotrigona pothieri.

$\begin{array}{lll}\text { Ruchers } & \begin{array}{l}\text { Formation } \\ \text { végétale }\end{array} & \text { Composition floristique }\end{array}$

RT Savane arborée

Savane à Loudetia simplex et Hyparrhenia sp avec Borassus aethiopicum, Bridelia ferruginea, Crossopteryx febrifuga, Cussonia barteri, Piliostigma thonningii, Terminalia glaucescens, Combretum spp, Vitex doniana, Cochlospermum, planchonii, Lippia sp, Annona senegalensis...

R1

Forêt galerie

Bord du

Bandama

Bord de

Chemin

Canopée dominée par Ceiba pentandra avec: Antiaris africana, Cynometra megalophylla, Dialium guineense, Erythrophleum
suaveolens, Lecaniodiscus cupanoides, Manilkara obovata, Mansonia altissima, Mimusops cummel; en sous-bois: Dalbergia oblongifolia, Diospyros soubreana, Dichrostachys sp, Drypetes floribunda, Grewia carpinifolia, Pancowia bijuga, Rothmannia whitfieldii; de grandes lianes arbustives: Cremaspora triflora, Flabellaria paniculata, Griphonia sp, Salacia sp, Strychnos sp

R2 Lisière forêt/ Savane (cf RT); Forêt (cfR1) avec en outre: Afzelia africana, savane lisière: Antidesma membranaceum, Bersamia paulinioides, Dalbergiella welwitchii, Entada mannii, Dracaena perrotteti, Elaeis guineense, Phcenix reclinata.

CfR1, avec comme espèces dominantes: Antiaris africana, Enythrophleum guineense; nombreuses lianes: Dioscorea minutiflora, Combretum racemosum, Smilax crossiana.

Cola gigantea, Detarium senegalense, Dialium guineense, Dracaena perrotteti, Holoptelea grandis, Holarhenia floribunda, Sterculia tragacantha; lianes: Adenia cissampeloides, lpomoea spp, Combretum spp 
- R3: un nid placé dans un tronc de Dialium guineense, au milieu de la galerie forestière, près d'un marigot et sous un couvert végétal très dense et extrêmement fermé;

- R4: 2 nids, placés sur un tronc couché de Pseudospondias microcarpa ayant créé une large trouée dans la galerie forestière du nord;

- R5: 2 nids, situés sur la station biologique, dans un milieu ouvert aménagé, comprenant des espèces arbustives et arborescentes de la savane et des espèces introduites.

\section{TECHNIQUES}

Les nids des Hypotrigones, situés dans les creux des branches et des troncs d'arbres (Darchen, 1985), sont nécessairement détruits lors de leur ouverture pour en extraire les produits de réserve. Les miels à partir desquels ont été faites les analyses proviennent de l'écoulement de l'ensemble des cellules à miel des ruches. Les rations alimentaires ont été extraites à partir de la totalité du couvain conservé dans l'alcool à $70^{\circ}$. Le pollen a été isolé, puis acétolysé à partir de $20 \mathrm{cc}$ de ces 2 substances (10 cc seulement dans le cas du miel provenant de l'échantillon R3), selon les méthodes de Louveaux et al $(1970,1978)$ et de Lobreau-Callen et Calien (1982). L'analyse pollinique de ces miels et des rations alimentaires traduit donc ainși la composition globale des réserves en miels. Par ailleurs, 1200 grains de pollens ont été comptés et identifiés pour chaque échantillon, de telle sorte que tous les taxons présents aient été rencontrés au moins une fois (Vergeron, 1964).

Les pollens stockés ont été prélevés cellule à cellule choisie au hasard, puis acétolysés. Le contenu relativement ho- mogène de ces cellules, 1 à 4 espèces, nous a conduits à identifier les espèces représentées et à les classer selon leur indice de dominance (variant de ++++ pour les plus abondants à $\varepsilon$ pour les plus rares). Une telle analyse peut paraître moins complète, les calcuis en pourcentages n'étant pas réalisés. S'il est difficile de définir, dans les conditions de l'expérience, l'âge des cellules de réserves, on peut en revanche dire que les cellules de couvain ont entre 1 jour et 38-39 jours, compte tenu du temps de développement de ces abeilles. Toutefois, l'àge de la construction des cellules n'implique pas pour autant que la gelée nourricière dont elles sont remplies soit contemporaine, les ouvrières pouvant utiliser, pour sa préparation, des réserves contenues dans la ruche depuis assez longtemps; c'est d'ailleurs ce que nous allons voir.

\section{RÉSULTATS}

L'ensemble des résultats analytiques est détaillé dans le tableau II selon la nature du matériel et son origine.

\section{Les miels}

Quelle que soit l'origine du miel étudié, on constate que le volume de pollen est relativement constant et s'élève à environ 0,2$0,3 \mathrm{ml}$. On remarque également que, dans l'ensemble des spectres, la diversité des taxons récoltés en quantité significative (> 0,5\%: 17 dicotylédones, 3 monocotylédones) est relativement pauvre par rapport à la richesse floristique environnante.

Si l'on examine l'échantillon témoin RT, originaire de la savane, 2 taxons seulement dominent très largement avec respectivement plus de $35 \%$. Dans le cas de 
Bridelia ferruginea $(37,7 \%)$ dont la floraison a lieu aux environs de février, il ne peut s'agir que d'un stockage provenant des floraisons de printemps, tandis que dans celui de Borassus aethiopicum $(36,4 \%)$, il pourrait s'y être adjoint quelques apports correspondant à la floraison plus ou moins continue de certains individus. Le pourcentage d'Entada mannii, en pleine floraison au moment de la prise de l'échantillon, est également relativement élevé $(17,2 \%)$ et laisse penser qu'il aurait pu être supérieur dans une récolte plus tardive. Par ailleurs, on remarque que les rares autres espèces privilégiées (Erythrophleum, Lecaniodiscus, type Loncocarpus) sont de type arbustif et lianescent de savane ou de lisière bien éclairée, à floraison printannière. Ainsi, le miel du rucher témoin représente un mélange de récoltes récentes et de récoltes anciennes datant de plusieurs mois.

Dans l'analyse du rucher R2, assez comparable dans son biotope secondaire, le transfert du nid n'apporte pas de modifications importantes pour les éléments les plus significatifs tels que Bridelia et Borassus. Les autres taxons bien représentés ne sont pas plus nombreux que dans RT et appartiennent très évidemment à des espèces de la lisière forestière particulièrement proche des nids et toujours bien ensoleillée: Lecaniodiscus, Entada, Ceiba, Lannea. Dans un milieu similaire, on constate donc que le comportement des abeilles est tout à fait comparable, se réduisant à la récolte du nectar sur quelques espèces végétales communes, proches des nids.

Lorsque les nids ont été placés en forêt (R1, R3), les spectres sont très différents. Ainsi, dans les récoltes de R3, situé dans un milieu fermé, particulièrement couvert et sombre, le pourcentage de pollen de Bridelia et de Borassus est réduit de moitié, alors que celui de Lecaniodiscus, pré- sent dans le sous-bois, augmente considérablement $(30,8 \%)$, de même que celui des Cypéracées de bord de marigot (20\%).

Placé au bord d'un chemin forestier, le nid $R 1$ donne un spectre pollinique dans lequel Borassus offre un pourcentage extrêmement réduit $(1,2 \%)$, alors que celui de Bridelia est, cette fois, au contraire plus élevé que celui du témoin. II convient de noter que, sur ce site, il existe une espèce forestière de Bridelia, différente de $B$ ferruginea très abondant en savane (Vuattoux, communication personnelle). Les autres pourcentages les plus significatifs correspondent à des espèces forestières, arborescentes ou lianescentes de la canopée, dont la floraison se situe soit avant la transplantation du nid (Erythrophleum), soit au moment de son ouverture (Entada, Fagara).

Dans le rucher $R 4$, les 2 nids étudiés donnent globalement des résultats similaires. Les pourcentages de Bridelia et de Borassus sont fortement réduits. En revanche, celui d'Adenia, liane à floraison estivale, abondante près du rucher, est particulièrement élevé $(79,6 \%$ et $95,9 \%)$.

Le dernier emplacement (R5) correspond à une zone ouverte sur laquelle il existe de nombreuses plantes introduites ou indigènes cultivées à floraisons abondantes et attractives. Cependant les résultats sont très variables d'un nid à l'autre : dans l'un des deux, les abeilles ont été spécialement attirées par les fleurs de Cassia $(87,7 \%)$ et dans l'autre, dont l'ouverture était orientée différemment, elles ont préféré butiner Erythrophleum (43,5\%) et Bridelia (21,3\%).

\section{Le pollen}

L'analyse des cellules à pollen donne, dans son ensemble, des spectres plus lar- 


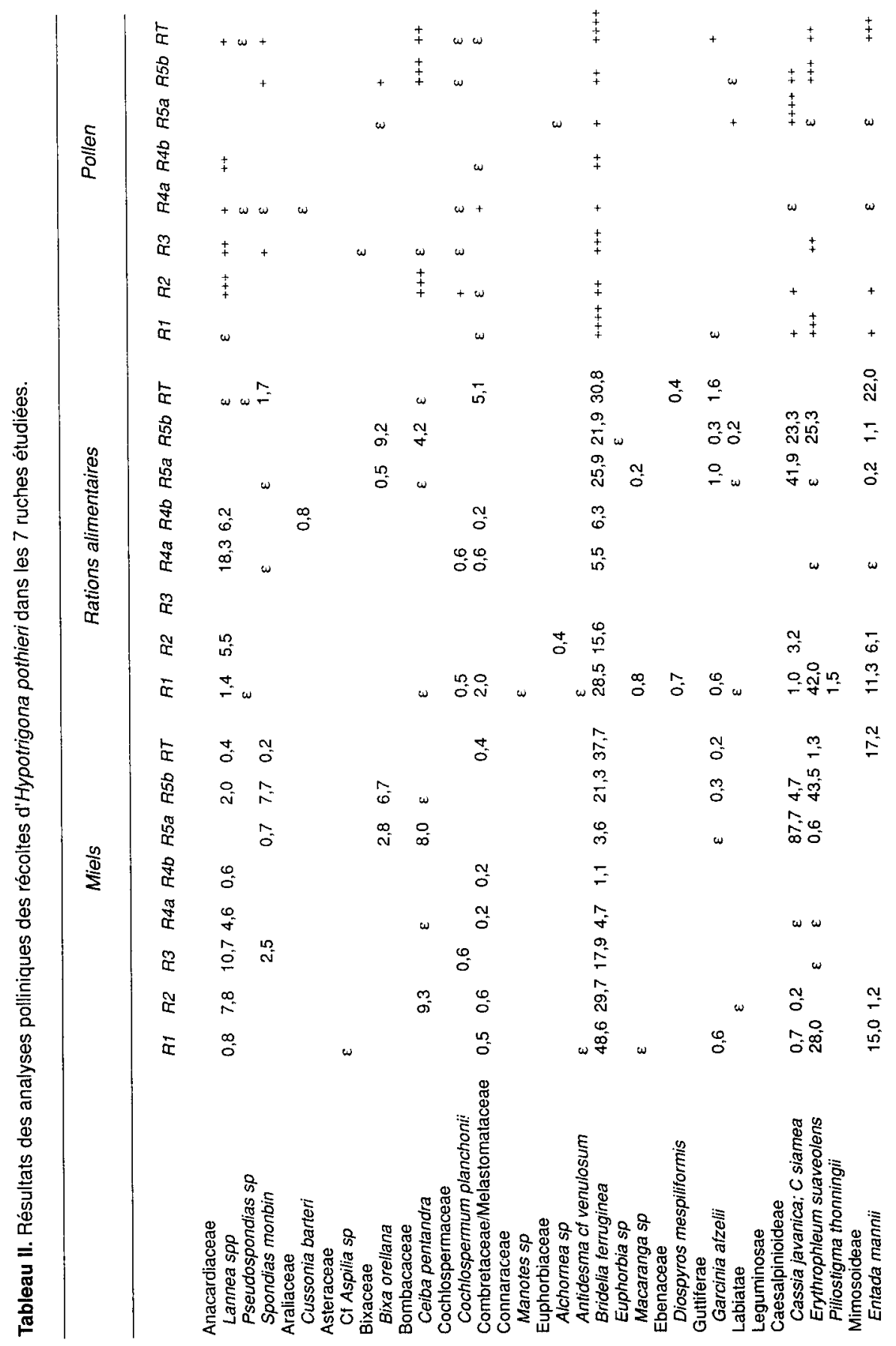








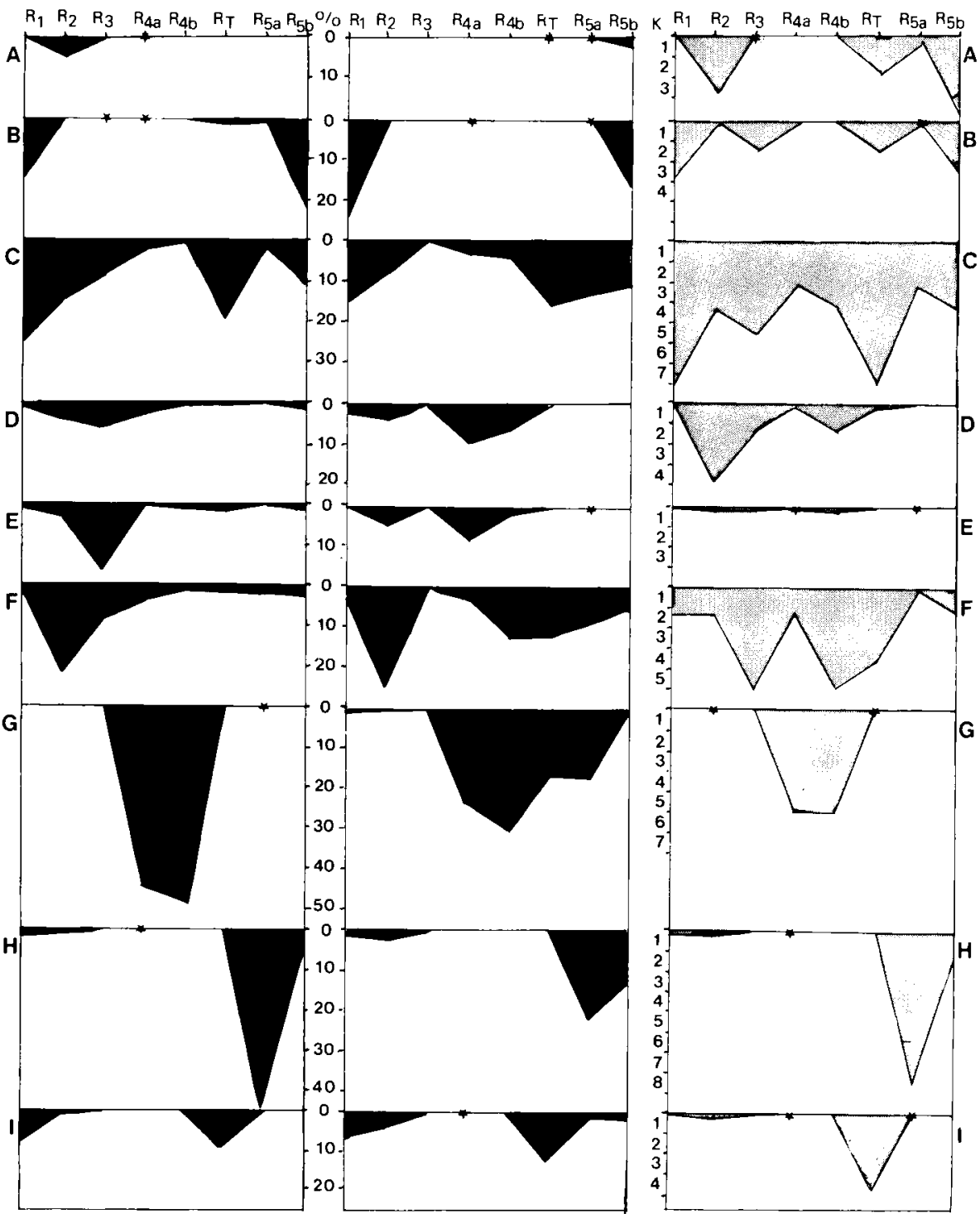

Fig 1. Diagrammes polliniques des principaux éléments récoltés par Hypotrigona pothieri. Dans les miels et les rations alimentaires, les quantités de pollens sont indiquées en pourcentages relatifs par rapport aux 1200 grains comptés. Dans les cellules à pollen, elles sont exprimées en classes. Lorsque les quantités sont très faibles $(<1 \%)$, elles sont indiquées par une étoile (*). $K=$ classes: $1,0,5-$ $12,5 \% ; 2,12,6-25 \% ; 3,25,1-37,5 \% ; 4,37,6-50 \% ; 5,50,1-62,5 \% ; 6,62,5-75 \% ; 7,75,1-87,5 \% ; 8$, 87,5-100\%. Floraisons de printemps: $A=$ Ceiba pentandra, $B=$ Erythrophleum suaveolens, $C=B r i-$ delia ferruginea, $\mathrm{D}=$ Lannea spp, $\mathrm{E}=$ Blighia sapida / Lecaniodiscus cupanoides. Floraisons plus ou moins continues: $\mathrm{F}=$ Borassus aethiopicum. Floraisons estivales: $\mathrm{G}=$ Adenia cissampeloides, $\mathrm{H}=$ Cassia javanica/C siamea, I = Entada mannii. 
gement diversifiés en taxons que ceux des miels. Toutefois, chaque cellule contient seulement 2 à 3 taxons dominants, récoltés à la même saison. De plus, chacune des pelotes qui les constituent ne renferme le pollen que d'une seule espèce végétale; ceci indique, qu'au cours d'un voyage, les abeilles butinent le pollen d'une seule espèce.

Si l'on considère les taxons dominants (fig 1), on voit que leur répartition est comparable à celle qui a été mise en évidence dans les miels. Malgré la diversité des espèces végétales, il existe donc le même type de choix de la part des abeilles lorsqu'elles récoltent le pollen et le nectar.

\section{Les réserves alimentaires des larves}

Ces réserves alimentaires sont constituées d'un mélange de miels et de pollens déposé dans les cellules à couvain où se nourrissent les larves qui deviennent adultes après un peu moins de 6 semaines. Dans la comparaison de l'analyse pollinique de ces cellules avec celles des miels ou du pollen, on retrouve en grande partie des pourcentages comparables des taxons récoltés. Cependant, on constate que les plantes dont la floraison n'a débuté qu'un mois environ avant l'ouverture de nids sont beaucoup moins bien représentées dans les réserves alimentaires. Ainsi, par exemple, dans le rucher R5, le pourcentage de Cassia est-il beaucoup moins élevé $(41,9 \%)$ que dans les miels ou les cellules à pollen. Les réserves alimentaires apparaissent donc comme essentiellement constituées par des récoltes datant de plusieurs mois et provenant, plus particulièrement des miels arrivés à maturité. Pour nourrir leurs larves, les abeilles utiliseraient donc, en priorité, les stocks de miels et de pollen anciens (Erytrophleum, Bridelia) tout en $y$ ajoutant du pollen frais provenant d'espèces en pleine floraison.
La cohérence des résultats mise en évidence dans l'analyse pollinique des réserves et des rations alimentaires nous permet de donner une interprétation globale de ces observations.

\section{DISCUSSION}

L'analyse générale de l'ensemble du contenu des nids d'Hypotrigona pothieri montre, en premier lieu, que ces abeilles sociales pratiquent un choix très restreint dans la végétation environnante, même à proximité de leur habitat. Sur un demicycle annuel, on relève au maximum une dizaine de taxons bien représentés dans leurs récoltes $(>0,5 \%)$. Comme l'ont déjà constaté Sommeijer et al (1983), ils peuvent correspondre à des plantes de floraison plus ou moins continue (Borassus), mais ils peuvent également représenter des plantes à floraison épisodiquement abondante, indigènes comme Erytrophleum, Bridelia, Entada, ou encore, cultivées comme Cassia siamea et Cassia javanica. Les Hypotrigones apparaissent donc relativement sélectives, puisqu'elles négligent totalement des espèces abondantes et en pleine floraison près de leurs nids (par exemple, Piliostigma). Cette sélectivité tendrait à prouver qu'elles participent réellement à la pollinisation des taxons privilégiés dans leurs récoltes, attirées par leurs inflorescences denses, composées de multiples fleurs odorantes et colorées (Faegri et Van der Pijl, 1979) mais surtout riches en nectar dont la qualité paraît déterminante (Baker et Baker, 1983; Frankie et Haber, 1983; Vogel, 1983). En outre, Baker et Baker (1975) ont montré que les nectars de plantes melittophiles renferment plusieurs substances autres que les sucres et, en particulier, certains alcaloïdes considérés comme répulsifs pour certaines abeilles (Thomson, 1986). 
Dans l'analyse d'un miel de Lamto (Vaquette, inédit), on a pu effectivement constater un très fort pourcentage d'alcaloïdes $(0,05 \%)$, sans toutefois pouvoir l'attribuer, par spectrographie, à une espèce précise du spectre pollinique. Ceci pourrait cependant expliquer la très forte attirance des Hypotrigones pour le genre Bridelia, les Euphorbiacées étant particulièrement riches en alcaloïdes.

Malgré l'aspect très sélectif de leur butinage, les Hypotrigones gardent un caractère d'opportunistes, si l'on considère que les espèces privilégiées restent généralement proches de l'environnement des nids. On peut donc dire que ces abeilles n'ont pas un véritable comportement de spécialistes, adaptées à une structure florale particulière, mais qu'elles sont susceptibles d'utiliser de nombreux types floraux pour subvenir aux besoins de la colonie (Heinrich, 1983). Si l'on constate que, dans les ruchers RT et R2, placés dans un même biotope, le contenu des miels révèle des pourcentages comparables pour les espèces privilégiées (Borassus, Bridelia), en revanche, dans les ruchers R1, R3, R4, R5, situés dans des milieux différents, les pourcentages respectifs de ces 2 espèces sont profondément modifiés. En particulier, le spectre du rucher $\mathrm{R} 4$, réimplanté dans un petit chablis forestier, traduit une chute considérable du pourcentage de ces 2 taxons, au bénéfice d'une espèce largement dominante, Adenia cissampeloides, généralement très peu représentée dans le contenu des miels des autres ruches. Dans ce cas, il ne peut s'agir d'une prédétermination à une structure florale particulière, mais seulement d'une espèce de substitution, abondamment fleurie à proximité des nids et directement exploitable d'un point de vue énergétique.

A l'inverse, dans le rucher R1, transporté en forêt-galerie, si l'on observe égale- ment une très forte diminution de Borassus, on remarque que le pourcentage de Bridelia a augmenté, en même temps que celui de quelques rares espèces forestières (Erytrophleum, Entada, Fagara). Les abeilles ont en fait remplacé le Bridelia de savane par l'une des 2 espèces forestières de la région, probablement plus attirées par la présence de substances riches en alcaloïdes, contenues également dans le nectar de Fagara, que par le type de structure florale.

Les résultats très différents que l'on observe dans les deux ruches R5 ( $a$ et $b$ ), situées au même endroit, nous conduisent à mettre en évidence un autre caractère du comportement des Hypotrigones. Le pourcentage très élevé de Cassia $(87,7 \%)$ dans R5a s'explique aisément par l'attirance bien connue des abeilles pour ce type de fleurs (Tischler, 1917); le pollen est extrait des anthères poricides par des abeilles munies de mécanismes adaptés (Van der Pijl, 1954; Michener, 1962; Wille, 1963; Buchmann et al, 1977) et, une fois dispersé, les Hypotrigones et les Dactylurines viennent le récolter en masse (observation de Lobreau-Callen). Dans le rucher $\mathrm{R} 5 \mathrm{~b}$, le pourcentage de Cassia est, au contraire, extrêmement faible, alors que celui de Bridelia ferruginea redevient significatif et, dans une moindre proportion, celui de Bixa orellana et de Spondias mombin. Ces 2 espèces étant en fait présentes dans le même périmètre que Cassia, mais dans une direction opposée, il semble que les préférences des abeilles, manifestées dans ces 2 nids, puissent être attribuées à l'orientation différente de l'ouverture des ruches. Dans le rucher R5b, le fort pourcentage d'Erytrophleum, fleuri bien avant l'installation des nids, ne peut représenter que des apports antérieurs à la transplantation et non consommés par les abeilles.

Enfin, dans le rucher R3 où les Hypotrigones sont pratiquement privées de lu- 
mière, les spectres polliniques montrent clairement qu'elles ont essentiellement vécu sur les réserves correspondant à des floraisons qui ont précédé le déplacement du nid, sans qu'elles aient pu emmagasiner suffisamment de nouvelles récoltes. Le manque de lumière semble donc perturber totalement leur comportement et les conduire ainsi à la disparition, malgré leur tentative de survie en exploitant les Cypéracées. L'absence de couvain confirme, semble-t-il, cette interprétation, les réserves alimentaires du nid étant devenues insuffisantes pour assurer la pérennité de la colonie.

\section{CONCLUSION}

La transplantation de nids d'une même espèce d'Hypotrigona dans plusieurs milieux différents permet, à travers l'analyse pollinique de leurs récoltes, de mettre en évidence certains aspects de leur comportement sur la végétation. Nos résultats palynologiques montrent que ces abeilles sociales peuvent butiner un grand nombre d'espèces en fleurs mais que, malgré leur caractère généraliste, elles demeurent cependant sélectives. Leur choix ne paraît nullement lié à une structure florale particulière, comme chez certaines abeilles sociales ou solitaires (Stebbins, 1970; Faegri et Van Der Pijl, 1979; Lobreau-Callen et Coutin, 1987), puisqu'elles substituent volontiers une des rares espèces privilégiées dans leurs récoltes habituelles, mais absente de leur nouvel environnement, par une autre espèce de type morphologique différent.

L'analyse comparative des récoltes de 2 nids placés dans un même rucher permet également de montrer l'importance de l'orientation de l'ouverture des nids qui semble être un facteur déterminant dans le choix des plantes exploitées par les Hypo- trigones, tandis que la lumière et l'éclairage restent sans doute les facteurs prédominants de leur comportement et même de leur survie.

D'autres modes d'expérimentation ont été utilisés pour étudier le comportement de butinage de certaines Trigones sl, mettant en lumière un schéma de sélectivité propre à chaque espèce et dépendant de la taille de la population de la colonie. L'observation que nous avons réalisée sur une seule et même espèce, transplantée dans des biotopes différents, permet de montrer que la complexité du comportement de ces abeilles sociales est également liée à un ensemble de facteurs exogènes, résultats qui devront être confirmés par une analyse portant sur un cycle annuel.

\section{REMERCIEMENTS}

Ce travail a été réalisé grâce au soutien de programme "Actions Biologiques" accordé au Laboratoire de phytomorphologie de l'EPHE par la direction de la recherche du MNE et à la participation financière du laboratoire Ecotrop, CNRS pour les missions sur le terrain. Nous remercions spécialement $R$ Vuattoux, directeur de la station de Lamto et $L$ Aké Assi, directeur du centre floristique d'Abidjan, pour leur assistance efficace.

\section{Summary - Some factors determining} foraging behaviour of Hypotrigona pothieri (Hym, Apidae) in the vegetation of Ivory Coast. Pollen analysis of crops from Hypotrigona pothieri Darchen (Hym, Apidae), social bees of the tribe Trigonini, were carried out on 7 hives installed in 5 different biotopes from woody savanna and from forest gallery in the region of Lamto. They were opened in July to August during the short dry season after a 5 month period. Their content is compared to 
one of a check hive in their original environment. The floristic inventory of the surroundings of these different hives is summarized in table I. The pollen extracted from honey, from pollen cells and from food supply was isolated and acetolysed. The quantity of pollen of each species from honey and from food supply is indicated in relative percentage of the total number of pollen grains for each sampling. In each case, 1200 pollen grains were counted and identified (Vergeron, 1964). For the pollen cells, the abundance ratio is indicated and the content of the cells are analyzed individually (table II).

The results indicate that, in spite of a rather extensive overall pollen spectrum, these social bees are generally opportunistic and fairly selective. They show a preference for a small number of plant species (dominant taxa, fig 1) always near the hives; the choice is independent of the type of flower structure but seems to be related to the presence of certain alkaloids in the nectars. These taxa are dominant taxa as much in the honey, as in the pollen cells or food supply. However, the quantities are different and the intermediate percentages noticed in the food supply show that they constitute a mixture of the two types of stores (honey and pollen). Pollen analysis of material from the two hives staying in the same biotope (R5a and $\mathrm{R} 5 \mathrm{~b})$ show that the choice of the gathered species is directly influenced by the orientation of the entrance of nests. However, when the Hypotrigones are displaced in forest gallery, the pollen content from the stores and from the food supply shows these bees survive only if they are situated in open areas (R1, R4). In the case where the hives were placed in dense undergrowth (R3), the bees survive with difficulty, their brood is not renewed and the colony finally dies out. Light seems indispensable to the good functioning of hives of Hypotrigona, as indicated by the association of their normal nesting biology with open areas, such as savannas.

Hypotrigona pothieri / foraging behaviour / pollen analysis / ecology / Apidae

\section{Zusammenfassung - Einige Faktoren, welche das Trachtverhalten von Hypotrigona pothieri (Hym, Apidae) in der Vegetation der Elfenbeinküste beeinflussen. Es wurden Pollenanalysen der Vorräte von Hypotrigona pothieri Darchen, Stachellose Bienen vom Tribus Trigonini, durchgeführt, die von 7 Kolonien nach Verpflanzung in 5 verschiedene} Biotope der Waldsavanne und des Galeriewaldes in der Gegend von Lamto gesammelt worden waren. Sie wurden 5 Monate nach ihrer Aufstellung in den Monaten Juli-August während der kleinen Regenzeit geöffnet; ihre Pollenvorräte wurden mit denjenigen eines Kontrollvolkes verglichen, das in seiner ursprünglichen Umgebung verblieben war. Die Florenzusammensetzung der verschiedenen Standorte ist in Tabelle I zusammengestellt.

Pollen aus Honig, aus Pollenzellen und aus Larvenfutter wurde isoliert und mit Säure behandelt (azetolysiert). Die Pollenmenge jeder Art aus dem Honig und aus dem Larvenfutter wird in dem relativen Prozentsatz und der Gesamtzahl der ausgezählten und bestimmten Pollenkörner (1 200) jeder Probe angegeben. Für die Pollenzellen ist die Häufigkeit für jede Zelle in Klassen angegeben (Tabelle II).

Die Ergebnisse zeigen, daß sich die Bienen, im allgemeinen Generalisten und Opportunisten, trotz eines sehr weiten Pollenspektrums relativ selektiv verhalten. Sie bevorzugen eine kleine Zahl von Pflanzenarten (dominierende Taxa in Abb 1), immer nahe der Kolonie; die Auswahl 
ist unabhängig vom Blütenbau, sie scheint aber von der Anwesenheit gewisser Alkaloide im Nektar abzuhängen. Diese dominierenden Arten finden sich sowohl im Honig wie in den Pollenvorräten und in dem Larvenfutter. Die Mengen sind jedoch verschieden, und die intermediäre Menge im Larvenfutter weist darauf hin, daß dieses aus den beiden Komponenten, Honig und Pollen, gemischt wurde.

Die Pollenanalyse von 2 Kolonien, die in demselben Biotop (R5a, R5b) aufgestellt waren, zeigt, daß die Auswahl der aufgesuchten Pflanzenarten direkt von der Orientierung des Flugloches abhängt. Bei Aufstellung im Galeriewald wiederum zeigte sich aus den Vorräten und der Larvenversorgung, daß die Kolonien nur bei freier Aufstellung gedeihen können (Weg, R1; Windbruch, R4); bei Aufstellung im dichten Unterholz (R3) hingegen konnten die Bienen nur schwer überleben, die Brut wurde unterbrochen und schließlich verschwanden sie. Auch Licht scheint für das Gedeihen der HypotrigonaKolonien unentbehrlich, deren Biologie unmittelbar mit dem offenen Milieu der Savanne verbunden ist.

\section{Hypotrigona pothieri / Trachtverhalten / Pollenanalyse / Ökologie / Apidae}

\section{RÉFÉRENCES}

Absy ML, Kerr E (1977) Algumas plantas visitadas para obtenço de polen por operarias de Melipona seminigra merillae en Manaus. Acta Amazonica 7, 309-315

Absy ML, Bezerna EB, Kerr WE (1980) Plantas nectariferas utilizadas por duas espécies de Melipona da Amazônica. Acta Amazonica 10, 271-281

Baker H, Baker I (1975) Studies of nectar constitution and pollinator - plant coevolution. In: Coevolution of Animals and Plants. (Gilbert
LE, Raven $\mathrm{PH}$, eds) University of Texas Press, Austin, 100-140

Baker H, Baker I (1983) Floral nectar sugar constituants in relation to pollinator type. In: Handbook of Experimental Pollination Biology (Jones CE, Little RJ, eds) Van Nostrand Reinhold, New York, 117-141

Buchmann SL, Jones CE, Colin JL (1977) Vibratile pollination of Solanum doulasii and $S$. xanti (Solanaceae) in Southern California. $J$ Biol 35 (1), 1-26

Cesar J, Menaut JC (1974) Analyse d'un écosystème tropical humide : la savane de Lamto (Côte-d'Ivoire). Bull Liaison Cher Lamto 2 ( $\mathrm{N}^{\circ}$ spécial)

Cortopassi-Laurino M, Ramalho M (1988) Pollen harvest by africanized Apis mellifera and Trigona spinipes in Sao Paulo. Botanical and ecological views. Apidologie 19 (1), 1-23

Darchen $R$ (1985) Les abeilles sociales d'une savane de Côte-d'Ivoire. Ecologie du genre Hypotrigona. Publ Sci Acc 7, 1-17

Devineau JL, Lecordier C, Vuattoux R (1984) Evolution de la diversité spécifique du peuplement ligneux dans une succession préforestière de colonisation d'une savane protégée des feux (Lamto, Côte-d'Ivoire). Candollea 39, 103-134

Engel MS, Dingemans-Bakels $F(1980)$ Nectar and pollen resources for stingless bees (Meliponinae, Hymenoptera) in Surinam (South America). Apidologie 2 (4), 341-350

Faegri K, Van Der Pijl L (1979) The Principles of Pollination Ecology. $3^{\mathrm{e}}$ éd, Pergamon Press, Oxford

Frankie GW, Haber HG (1983) Why bees move among mass-flowering neotropical trees. In: Handbook of Experimental Pollination Biology (Jones CE, Little RJ, eds) Van Nostrand Reinhold, New York, 360-372

Frankie GW, Haber WA, Opler PA, Bawa KS (1983) Characteristics and organization of the large bee pollination system in the Costa Rica dry forest. In: Handbook of Experimental Pollination Biology (Jones CE, Little RJ, eds) Van Nostrand Reinhold, New York, 411447

Heinrich $B(1983)$ Insect foraging energetics. In: Handbook of Experimental Pollination Biology (Jones CE, Little RJ, eds) Van Nostrand Reinhold, New York, 187-214 
Hubbell SP, Johnson LK (1977) Competition and nest- spacing in a tropical stingless bee community. Ecology 58, 949-963

Hubbell SP, Johnson LK (1978) Comparative foraging behavior of six stingless bees exploiting a standardized resources. Ecology 59, 1123-1136

Iwama S, Melhem TS (1979) The pollen spectrum of the honey of Tetragonisca angustulata Latreille (Apidae, Meliponinae). Apidologie 10, 275-295

Jaffre T, Namur Ch de (1983) Evolution de la biomasse végétale épigée au cours de la succession secondaire dans le sud-ouest de la Cóte-d'Ivoire. Acta Oecol, Oecol Plant (4) 18, 259-272

Johnson LK, Hubbell SP (1974) Aggression competition among stingless bees: field studies. Ecology 55, 120-127

Johnson LK, Hubbell SP (1975) Contrasting foraging strategies and coexistence of two bee species on a single resource. Ecology 56, 1398-1406

Kleinert-Giovannini A, Imperatriz-Fonseca VL (1987) Aspect of the trophic niche of Melipona marginata marginata Lepeletier (Apidae, Meliponinae). Apidologie 18 (1), 69-100

Lamotte M (1978) La savane préforestière de Lamto, Côte-d'Ivoire. In: Problèmes d'écologie : structure et fonctionnement des écosystèmes terrestres (Lamotte $M$, Bourlière $F$, eds) Masson, Paris 231-311

Le Thomas A, Lobreau-Callen D, Darchen B, Darchen R (1988) Analyse comparative des ressources polliniques et des stratégies de butinage de trois espèces de trigones sl en Côte-d'Ivoire. Inst Fr Pondichery Trav Sec Sci Tech 25, 345-354

Lobreau-Callen D, Callen G (1982) Quelle est la composition pollinique d'un miel exotique ? Bull Soc Versail Sci Nat Sér 4, 9 (4), 70-85

Lobreau-Callen D, Coutin R (1987) Ressources florales exploitées par quelques Apoïdes des zones cultivées en savane arborée sénégalaise durant la saison des pluies. Agronomie 7 (4), 231-246

Lobreau-Callen D, Darchen R, Le Thomas A (1986) Apport de la palynologie à la connaissance des relations Abeilles/Plantes en savanes arborées du Togo et du Bénin. Apidologie 1 (2), 211-217
Louveaux J, Maurizio A, Vorwohl G (1970) Commission internationale de botanique apicole de I'IUBS; les méthodes de mélissopalynologie. Apidologie 1 (2), 211-217

Louveaux J, Maurizio A, Vorwohl G (1978) Methods of melissopalynology. Bee World 59 (4), 139-157

Menaut JC (1973) Aperçu quantitatif sur les formations ligneuses des savanes de Lamto. CR Colloque PBI, Ann Univ Abidjan Sér E, 6 (2), 19-23

Michener CD (1962) An interesting method of pollen collecting by bee from flowers with tubular anthers. Rev Biol Trop 10 (2), 167-175

Roubik DW (1978) Competitive interactions between neotropical pollinators and africanized honey bees. Science 201, 1 030-1 032

Roubik DW (1979) Africanized honey bees, stingless bees and the structure of tropical plantpollinator communities. In: Proc IVth int Symp on Pollination. Md Agric Exp Sta Misc Publ 1 (Cron D, ed) College Park, Maryland, 403-417

Roubik DW (1980) Foraging behavior of competing africanized honeybees and stingless bees. Ecology 61, 836-845

Roubik DW (1981) Comparative foraging behavior of A mellifera and Trigona corvina (Hym, Apidae) on Baltimora recta (Compositae). Rev Biol Trop 29, 177-183

Roubik DW (1982) Ecological impact of africanized honeybees on native neotropical pollinators. In: Social insects in the Tropics (P Jaisson, ed) Univ Paris-Nord, 233-247

Roubik DW, Moreno JE, Vergara C, Wittmann D (1986) Sporadic food competition with the african honey bees: projected impact on neotropical social bees. J Trop Ecol 2, 97-111

Schmidt W (1973) Vegetationskundliche Untersuchungen im Savannenreservat Lamto (EIfenbeinküste). Vegetatio 28 (3-4), 145-200

Sommeijer ML, De Rooy GA, Punt W, De Bruijn LLM (1983) A comparative study of foraging behavior and pollen resources of various stingless bees (Hym., Meliponinae) and honey bees (Hym., Apinae) in Trinidad, WestIndies. Apidologie 14 (3), 205-224

Stebbins GL (1970) Adaptative radiation of reproductive characteristics in Angiosperms, I. Pollination mechanisms. Annu Rev Ecol Syst $1,307-326$ 


\section{Comportement de butinage}

Thompson JN (1986) Pattern in coevolution. In: Coevolution and Systematics (Stone AR, Hawksworth DL, eds) The Systematics Association, Clarendon Press, Oxford, Special vol, 32, 119-143

Tischler W (1917) Pollenbiologische Studien. $Z$ Bot $9,417-488$

Van Der Pijl L (1954) Xylocopa and flowers in the tropics 1-111. Proc $K$ Ned Ak Wet Ser C, 57, 413-423; 541-562

Vergeron P (1964) Interprétation statistique des résultats en matière d'analyse pollinique des miels. Ann Abeille 7 (4), 349-364

Vogel S (1983) Ecophysiology of zoophilic pollination. In: Encyclopedia of Plant Physiology,
New Series (Lange OL, Nobel PS, Osmond $\mathrm{CB}$, Ziegler $\mathrm{H}$, eds) Springer Verlag, Berlin 12 C, Physiological Plant Ecology III, 560624

Vuattoux R (1976) Contribution à l'étude de l'évolution des strates arborées et arbustives dans la savane de Lamto (Côte-d'Ivoire). Ann Univ Abidjan, Sér C (Sciences) 12, 35-63

Wille A (1963) Behavioral adaptation of bees for pollen collecting from Cassia flowers. Rev Biol Trop 11 (2), 205-210

Wille A, Orozco $E$ (1975) Observation on the founding of a new colony by Trigona cupira (Hym.: Apidae) in the Costa Rica. Rev Biol Trop 22 (2), 253-287 